\title{
Expression of inflammatory markers and NMDA receptors in the peripheral blood of stroke patients
}

\author{
ABOLFAZL AZAMI TAMEH ${ }^{1, \text { A, D, G }}$, BAHAREH BAHMANI 1, 2, B, c, E, F, MOHAMMAD KARIMIAN ${ }^{3, \text { D-F}}$, \\ ORCID ID: 0000-0003-2938-8902 \\ EBRAHIM KOUCHAKI', B, D, HAMIDREZA TALARI'1, B, D, CORDIAN BEYER ${ }^{4, A, D, ~ E}$ \\ ${ }^{1}$ Anatomical Sciences Research Center, Kashan University of Medical Sciences, Kashan, Iran \\ ${ }^{2}$ Department of Biotechnology, Iranian Research Organization for Science and Technology (IROST), Tehran, Iran \\ ${ }^{3}$ Department of Molecular and Cell Biology, Faculty of Basic Sciences, University of Mazandaran, Babolsar, Iran \\ ${ }^{4}$ Institute of Neuroanatomy, Faculty of Medicine, RWTH Aachen University, Aachen, Germany
}

A - Study Design, B - Data Collection, C - Statistical Analysis, D - Data Interpretation, E - Manuscript Preparation, F - Literature Search, G - Funds Collection

Summary Background. Identifying early molecular markers in ischemic stroke could help in reducing acute adverse effects of brain tissue after recanalization of clogged blood vessels.

Objectives. In this study, we evaluated the gene expression levels of glutamate $\mathrm{N}$-methyl-D-aspartate receptor (NMDAR) subunits (NR2A and NR3A) and inflammatory markers, as well as some oxidative stress markers in the peripheral blood of ischemic patients 24 $\mathrm{h}$ and $72 \mathrm{~h}$ after admission to the hospital.

Material and methods. 38 patients with ischemic stroke and 29 non-stroke individuals were included in the study. Prognosis and longterm outcomes of patients were evaluated using infarct area measurement from computed tomography scans and the Scandinavian neurological stroke scale. To identify serum biomarker changes, malondialdehyde (MDA) and NO (nitric oxide) levels were measured. A quantitative real-time PCR protocol was performed in order to evaluate the gene expression changes of NR2A, NR3A, TNF- $\alpha$ and interleukin-6 in selectively-isolated peripheral lymphocyte.

Results. Ischemic stroke induced a massive loss of lymphocytes (as determined by total lymphocyte RNA levels). The mRNA levels of $N R 2 A$ and NR3A were also significantly decreased $24 \mathrm{~h}$ and $72 \mathrm{~h}$ after admission to the hospital. We also observed a down-regulation of TNF- $\alpha$ and IL- 6 gene expression during the first day after admission. The serum level of NO showed a reduction from day one to day three, while MDA increased on day one of admission.

Conclusions. Changes in inflammatory cytokines following an ischemic attack could stimulate glutamate receptors in peripheral lymphocytes. As preliminary findings, this phenomenon could be considered as a prognostic and therapeutic tool in ischemic stroke management.

Key words: ischemic stroke, N-methyl-D-aspartate receptors, blood, lymphocytes, inflammation.

Tameh AA, Bahmani B, Karimian M, Kouchaki E, Talari H, Beyer C. Expression of inflammatory markers and NMDA receptors in the peripheral blood of stroke patients. Fam Med Prim Care Rev 2021; 23(1): 75-80, doi: https://doi.org/10.5114/fmpcr.2021.103160.

\section{Background}

Stroke is one of the leading causes of disability and death worldwide [1]. Most survivors suffer from dysfunctions in daily activities. Interruption in local blood supply during the ischemic phase, especially in the middle cerebral artery, causes several pathophysiological events, including acute necrosis, time-delayed neuroinflammation, neurotransmitter imbalance and brain tissue edema, triggering a stepwise degeneration (apoptosis) of neural tissue and networks, which ultimately worsen a patient's sensory and motor symptoms along with cognitive impairment. In addition to imaging techniques, such as computed tomography (CT) and magnetic resonance imaging (MRI), there are several brain and blood-associated hallmarks which could improve clinical diagnosis and prognosis of stroke [2]. It is necessary to understand molecular events related to the pathophysiology of ischemic stroke, like excitotoxicity, oxidative stress, inflammation and apoptosis, in order to improve clinical treatment in the future $[2,3]$. Ischemic stroke occurs after interruption of blood supply in the brain, which leads to neuronal tissue deprivation of glucose and oxygen [4]. These conditions cause biochemical and pathological alternations, like pro- inflammatory cytokine production and glutamate excitotoxicity, which appear simultaneously in the brain and peripheral blood and finally lead to the activation of the immune system [5]. Glutamate, a key stimulatory neurotransmitter in the mammalian central nervous system (CNS), is critically involved in the control of programmed cell death, and therefore, glutamate-mediated neurotoxicity could promote ischemia-dependent neuronal apoptosis [6]. Two types of glutamate receptors, such as ionotropic and metabotropic, are mainly associated with glutamate function. Activation of ionotropic N-methyl-D-aspartate receptor (NMDA) glutamate receptors results in the transfer of calcium ions into neurons, which causes the activation of apoptotic pathways [7]. Besides neurons, some NMDAR family members, like NR2 subunits, are found in other non-neuronal cell types, including blood cells [6], where they may regulate the activity of these immune cells [8]. NMDAR is composed of several subunits that denominate NMDAR1 (NR1), NR2A-D, NR3A and NR3B. Previous studies have revealed that NR1 and NR2 subunits are particularly expressed in human peripheral blood lymphocytes $[9,10]$ and further demonstrated that the expression pattern of NMDAR subunits may influence distinct disease states [9]. 


\section{Objectives}

This study was conducted to investigate possible alterations of the gene expression of NMDAR subunits (NR2A and NR3A) in peripheral blood lymphocytes as taken from stroke patients 24 $\mathrm{h}$ or $72 \mathrm{~h}$ after admission to the hospital, which is interpreted as the stroke onset. The present paper also investigated known inflammatory markers in study samples, including pro-inflammatory and oxidative stress cytokines and genes.

\section{Materials and methods}

\section{Subjects}

Control samples were obtained from 29 people admitted to the hospital's Lab for a routine check without neurological disorders or infections (18 women and 11 men; mean age 59 years). 38 patients with symptoms of stroke admitted within the first $6 \mathrm{~h}$ to the stroke unit of Shahid Beheshti University Hospital, Kashan, were recruited into the study and compared with age-matched non-stroke control subjects. These patients had their first acute ischemic stroke. Patients with hemorrhagic stroke, infectious diseases or cancer were not included in the study in order to avoid the confounding influence on inflammatory markers. Sample collection took 3 months. Ischemic stroke was clinically diagnosed by CT scans (Asteion, Toshiba) and neurological evaluations. The whole protocol was approved by the local ethics committee. Patients were admitted to the neurology ward and were managed according to evidence-based stroke strategies. Anti-platelet drugs were given to all patients with an obvious infarct detected by brain CT scan in most cases. Stroke severity was scored on the first day by a neurologist using the Scandinavian Neurological Stroke Scale (SNSS) with some modifications evaluating: consciousness, orientation, speech and verbal communication, eye movement, facial palsy, gait, power in arm, hand and leg. The score range was between 2 (maximum deficit) and 51 (minimum deficit) from a total score of 58. Body temperature and blood pressure were measured, and basic hematology, blood chemistry and a brain CT scan were performed on all patients. A non-enhanced brain CT scan was performed for infarct surface area calculation, infarct subtype analysis and infarct topography. Maximum surface area of the infracted region was measured by a routine measurement program (Asteion, Toshiba). Patient characteristics are summarized in Table 1.

\begin{tabular}{|l|l|l|}
\hline \multicolumn{3}{|l|}{ Table 1. Baseline characteristics of stroke cases and controls } \\
\hline Parameter & Controls $(n=29)$ & Cases $(n=38)$ \\
\hline Age (mean \pm SD) & $58 \pm 5$ & $59.7 \pm 5.7$ \\
\hline Gender (male/female) & $11 / 18$ & $12 / 26$ \\
\hline Infract size $\left(\mathrm{mm}^{2}\right)$ & NA & 423 \\
\hline Localization of infarction & NA & $\begin{array}{l}12 \text { MCA } \\
9 \text { PCA } \\
17 \text { lacunar }\end{array}$ \\
\hline CRP $(\mathrm{mg} /$ L) & & $29.6 \pm 1.4$ \\
\hline Lymphocyte (\%) & $15 \pm 0.8$ & $32.5 \pm 2.7$ \\
\hline
\end{tabular}

12 patients had ischemia in the middle cerebral artery territory (MCA) 9 in the posterior cerebral artery territory (PCA) and 17 had lacunar as diagnosed from CT scans. NA - not applicable. CRP - C-reactive protein.

$3 \mathrm{ml}$ of blood from patients was collected after $24 \mathrm{~h}$ and $72 \mathrm{~h}$ in tubes containing the same volume of PBS/EDTA. $3 \mathrm{ml}$ Lympholyte-H (Cedarlane, Canada) was added to the blood and centrifuge at $800 \mathrm{~g}$ for 20 minutes at room temperature according to the manufacturer's protocol. The lymphocyte layer and serum were collected separately and immediately frozen at $-80^{\circ} \mathrm{C}$ until use.

\section{Malondialdehyde assay}

Malondialdehyde (MDA) levels were determined using an MDA assay kit (NWLSS, Canada) according to the manufacturer's protocol. A $10 \mu \mathrm{l}$ BHT reagent was added to the centrifuge vials, and $250 \mu \mathrm{l}$ serum was then added to the vials. Next, $250 \mu \mathrm{L}$ acid reagent was added. In the next step, a $250 \mu$ I TBA reagent was added to the vials and mixed. Samples were incubated for $1 \mathrm{~h}$ at $60^{\circ} \mathrm{C}$ and centrifuged at $10,000 \times \mathrm{g}$ for 3 min. Finally, samples were measured at $532 \mathrm{~nm}$ using a spectrophotometer (APEL, Japan).

\section{Quantitation of nitric oxide}

Nitric oxide (NO) is normally a short-lived molecule with a half-life of a few seconds. In the blood, it is converted into other molecules, like nitrate and nitrite, which are stable molecules, so in this investigation, NOx (as the final product of NO) was evaluated. NO was measured in the serum by quantifying the final products of its reactions, nitrates and nitrites using a colorimetric assay. The nitrates in the serum were converted into nitrites by incubation with nitrate reductase and NADPH at room temperature for $2 \mathrm{~h}$. The levels of nitrites were then measured with the use of the Griess reaction by adding $100 \mu \mathrm{L}$ of Griess reagent ( $1 \%$ sulfanilamide and $0.1 \%$ naphthyl ethylenediamine in $5 \%$ phosphoric acid) to each plate and gently shaken for 20 min at room temperature. The addition of the Griess reagent results in a colorimetric product, which can be measured at $540 \mathrm{~nm}$. For that, standard curves were generated by nitrate dilutions.

\section{RNA extraction and reverse transcription}

For gene expression studies, RNA extraction from lymphocytes was done using the NucleoSpin DNA/RNA/Protein kit according to the manual (Macherey-Nagel, Germany). Total RNA was measured with a Biophotometer (Eppendorf, Germany) and calculated in $\mu \mathrm{g} / \mathrm{mL}$, and $0.5 \mu \mathrm{g}$ RNA was reverse transcribed using M-MLV with random primers at a final volume of $20 \mu \mathrm{L}$ (Invitrogen, Germany).

\section{Quantitative RT-PCR}

An SYBR green PCR master mix was used to amplify the desired fragments using specific primers including NR2A (Fwd: TGTGAAGAAATGCTGCAA GG, Rev: ACTGCCCGTTGATAGACC AC), NR3A (Fwd: CCACTCCACTGGACAATG T, Rev: GGTTTGATGGCCACTGTT CT), IL-6 (Fwd: TACCCCCAGGAGAAGATT CC, Rev: TTTTCTGCCAGTGCCTCT TT) TNF- $\alpha$ (Fwd: TCCTTCAGACACCCTCAA CC, Rev: AGGCCCCAGTTTGAATTC TT), GADPH (Fwd: GAGTCAACGGATTTGGTC GT, Rev: TTGATTTTGGAGGGATCT CG).

PCR reactions were carried out on MicroAmp ${ }^{\circledR}$ Optical 96well reaction plates (Applied Biosystems, USA) containing a $5 \mu \mathrm{l}$ SensiMix master mix (Bioline, England), 0.5 of each primer (10 $\mu \mathrm{M}$ stock), $2 \mu \mathrm{l}$ water and $2 \mu \mathrm{l}$ of 1:10 diluted cDNA. Gene expression was measured using RT-PCR technology (Applied Biosystems, USA) and the standardized protocol as described previously. For calculating gene expression, the Relative Standard Curve method was applied, where results were calculated from the ratio of the target gene quantity to the reference gene quantity [11]. Glyceraldehyde 3-phosphate dehydrogenase (GAPDH) was used as a housekeeper gene. The melting curve of the PCR products of each gene was checked to control the specificity of the $P C R$ reaction.

\section{Data analysis}

Data was statistically analyzed using SPSS 16.0 software (SPSS Inc., USA). For comparison between groups, one-way ANOVA and post-doc Tukey tests were used to determine the overall significance between groups. To calculate the correlation between the interleukin- 6 (IL-6) levels and size of a brain lesion, 
Spearman's rank-order correlation method was used. Data were given as means \pm SEM. $P$-values less than 0.05 were considered as statistically significant.

\section{Results}

In the current study, we analyzed the gene regulation of ionotropic NMDARs and inflammatory markers after ischemic stroke in human peripheral blood samples. Information about SNSS scoring, infarct size and patients, such as gender and age, is given in Table 1. CT images were examined to determine the type, site and volume of the infarcted brain area. The median SNSS score of stroke patients was 39 (ranging from 2-51), and the median infarct size was $423 \mathrm{~mm}^{2}$ (ranging from 65-1,132 $\mathrm{mm}^{2}$ ). Alterations in inflammatory markers, NMDAR subunits and lymphocyte levels in peripheral blood were analyzed by real-time PCR analysis and ELISA. Total lymphocyte-associated RNA levels (after selective isolation of lymphocytes from the same volume of blood) were significantly reduced compared to non-stroke patients one day after stroke $(p<0.05)$ (Figure 1$)$. This significant effect was no longer detectable three days after stroke, although the levels were still slightly less than in control cases. As mentioned in Table 1, the controls were non-stroke individuals matched to the patients by gender and age.

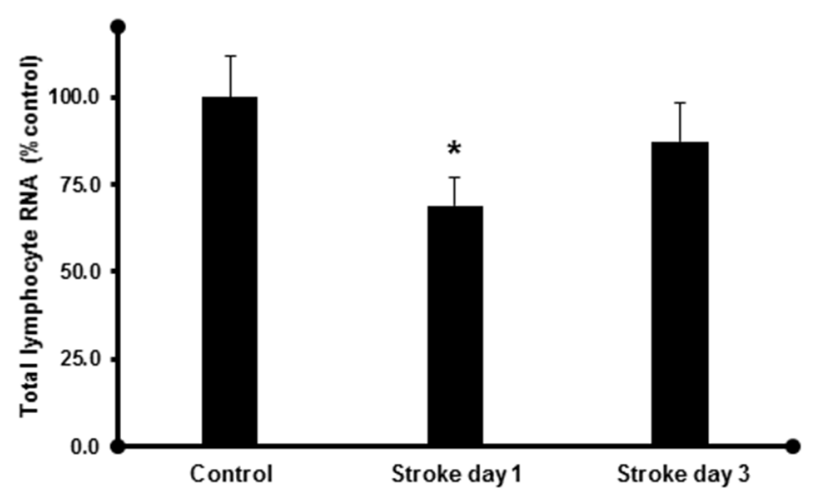

Figure 1. The graph shows a comparison of total lymphocyte RNA between control and ischemic stroke patients

Lymphocytes were isolated from the same amount of blood, and RNA isolation was done in all samples identically. ${ }^{*} p<0.05$ and compared to controls.

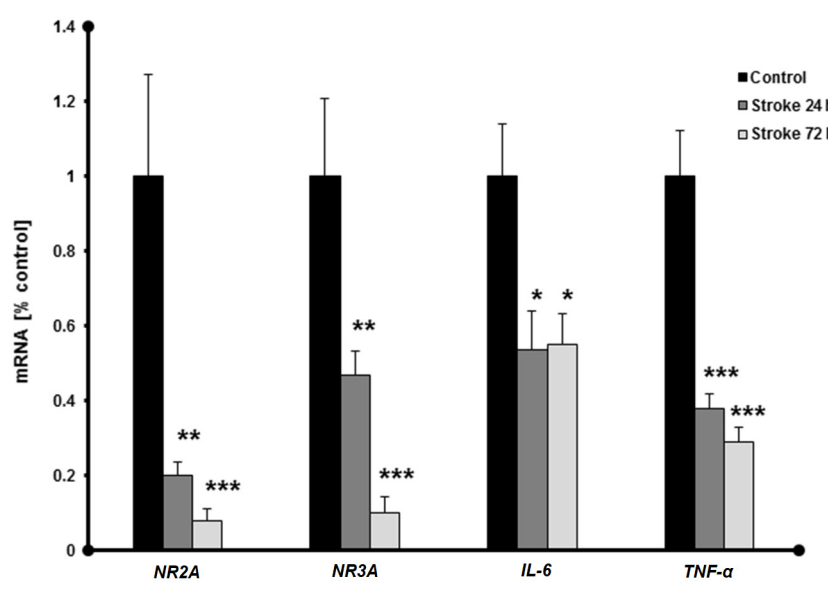

Figure 2. Data shows the gene expression of NMDAR subunits and inflammatory factors in peripheral blood lymphocytes $24 \mathrm{~h}$ and $72 \mathrm{~h}$ after stroke by real time PCR.

${ }^{*} p<0.05, * * p<0.01, * * * p<0.001$.
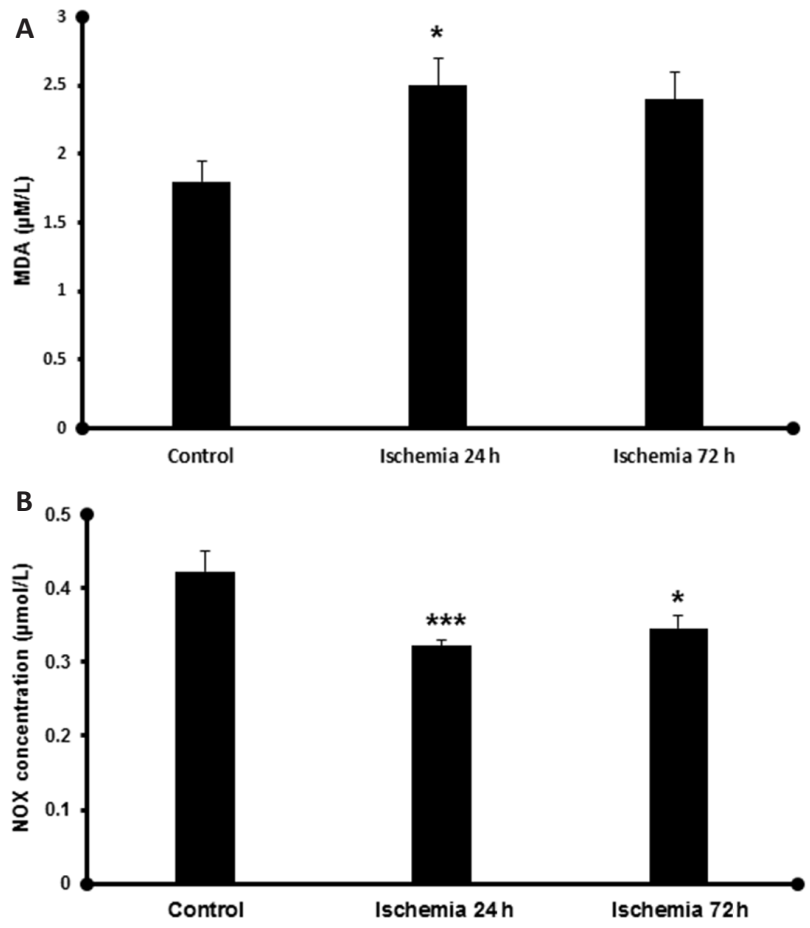

Figure 3. Data shows serum concentrations of malondialdehyde (MDA) and nitric oxide products (NOX) in control and stroke patients. A) Serum MDA levels within $24 \mathrm{~h}$ and $72 \mathrm{~h}$ of ischemic stroke onset. Levels of MDA significantly increased $24 \mathrm{~h}$ after stroke onset. B) Serum NOX levels $24 \mathrm{~h}$ and $72 \mathrm{~h}$ after ischemic stroke.

The level of NOX dramatically decreased $24 \mathrm{~h}$ and $72 \mathrm{~h}$ after stroke onset. ${ }^{*} p<0.05,{ }^{* * *} p<0.001$.

Gene expression of inflammatory factors and NMDAR subunits was then evaluated in peripheral blood lymphocyte preparations. As shown in Figure 2, a significant down-regulation of $N R 2 A$ and NR3A was observed in stroke samples compared to control human samples. Expression levels of NR2A and NR3A massively decreased during the initial day after stroke onset $(p<$ $0.01)$ and further declined thereafter until day three $(p<0.001)$. A similar significant decline of TNF- $\alpha$ and IL- 6 expression was detected after $24 \mathrm{~h}(p<0.001, p<0.05$, respectively). In addition, isolated serum was subjected to MDA and NOX analyses $24 \mathrm{~h}$ and $72 \mathrm{~h}$ after stroke onset. Serum MDA levels of ischemic brain patients was significantly $(p<0.05)$ elevated $24 \mathrm{~h}$ after stroke onset and returned to basal levels on day three after stroke (Figure $3 \mathrm{~A}$ ). In contrast, NOX concentrations were significantly reduced in stroke patients after $24 \mathrm{~h}(p<0.001)$ and $72 \mathrm{~h}$ $(p<0.05)$ compared to controls (Figure 3B).

\section{Discussion}

Occlusion of brain vessels is etiological for ischemic stroke, and acute hypoxia triggers a sequence of pathophysiological processes beginning immediately after ischemia and continues for hours or days. Primary neuronal damage mainly occurs within the first few hours and involves the breakdown of the blood-brain barrier and inflammatory responses in the brain, as well as in the peripheral circulation [12]. Our study investigated the consequence of ischemic stroke in human patients on the changes of glutamate receptors and expression of inflammatory molecules in peripheral blood. The primary outcome of this analysis was that the expression of NR2A and NR3A subunits, as well as IL- 6 and TNF- $\alpha$, was significantly reduced in lymphocytes $24 \mathrm{~h}$ and $72 \mathrm{~h}$ post stroke. MDA and NOX concentrations revealed opposite reactions, with MDA increasing and NOX decreasing, respectively. 
Emerging evidences suggest that peripheral white blood cells and secreting inflammatory mediators play a key role in the pathophysiology of stroke [13]. Previous studies reported that rapid lymphocytopenia is mainly found in T lymphocytes, which is the result of induced-apoptosis by increased cortisol and catecholamine plasma levels, which are known as stress mediators [14]. Increasing these mediators in the blood can increase apoptotic splenocyte and decrease the number of peripheral blood lymphocyte causing immunosuppression. In animal models, apoptosis of lymphocytes is observed in the spleen and thymus, and it is claimed that the number of apoptotic lymphocytes rises $12 \mathrm{~h}$ after middle cerebral artery occlusion (MCAO) [14]. This phenomenon could be an explanation as to why the risk of a systemic infection is elevated due to this immunosuppression several days after cerebral ischemia [15, 16]. In addition to a reduction of the number of immune cells in peripheral blood, decreasing TNF- $\alpha$ and IL- 6 levels occurred after stroke [17]. Our real-time PCR data supports this view and shows that the capacity of peripheral lymphocytes to produce TNF- $\alpha$ and IL-6 is impaired. In the brain, hampered neural cells and activated inflammatory cells both produce cytokines, such as IL-16, TNF- $\alpha$ and IL-6 [18]. These factors may act in the brain in a paracrine way within damaged tissue rather than being the source of cytokines in the periphery [19]. Along with our results, which demonstrate a reduction of TNF- $\alpha$ levels $24 \mathrm{~h}$ and $72 \mathrm{~h}$ after stroke, a previous study revealed that the secretion of TNF- $\alpha$ was significantly decreased in blood after MCAO [14]. Urra et al. described that the capacity of stimulated monocytes to release $T N F-\alpha$ is decreased in patients with acute stroke [19]. The relationship between peripheral IL-6 levels and stroke is not clear, although it has been reported that $I L-6$ has both neurotropic and anti-inflammatory effects, which could be essential after ischemia. Thus, IL- 6 may play a role in the induction and propagation of inflammatory responses in the brain [20], and our data showed that an attenuation of $I L-6$ expression is evident in the blood $24 \mathrm{~h}$ after stroke.

Our study also revealed that: (I) lymphocytes express NR2A and $N R 3 A$ subunits but not $N R 2 B$ and $N R 3 B$ subunits (data not shown); (II) the expression levels of both receptor subunits are reduced after stroke during the first three days. In addition to the expression of NR2A and NR3A NMDAR subunits in the brain, they are also expressed in peripheral blood. Both these NMDARs have been detected in human and rat lymphocytes [9]. It seems clear that NMDAR-mediated excitotoxicity takes part in promoting neuronal damage after stroke. Synaptic and extrasynaptic NMDARs have different functions in excitotoxic neuronal death [21]. NR2A containing NMDARs promotes neuronal survival rather than excitotoxicity $[18,22]$. As they are more associated with NR2B subunits, those receptors located on dendritic spines are functionally linked to perturbing the cytoskeletal protein F-actin but had no effect on neuronal death [18, 19]. In addition, synaptic NMDAR activity boosted antioxidant defenses, such as thioredoxin activity; however, extra-synaptic NMDAR action is a part of the glutamate-mediated neurotoxic cascade [23]. mRNA expression of $N R 2 A / 2 B$ receptor subunits appears to be decreased in rats with stroke within the first 24 $\mathrm{h}$ [24]. In a cellular model of excitotoxicity, levels of the NR2A subunit decreased rapidly with the induction of activated NMDARs. Regulation of NR2A during excitotoxicity is dependent on calpain activity and requires a calcium influx. This protease cleaves the C-terminal region in the NR2A subunit, producing a fragmented subunit lacking sequences which are important for survival, signaling cascades in the post-synaptic membrane [25]. Selective enhancement of NR2A-containing NMDARs may constitute a promising therapy for stroke [21]. Similarity, the $N R 3 A$ subunit plays a neuroprotective role in the CNS. Knocked out mice for NR3A increased the 2, 3, 5-triphenyltetrazolium chloride (TTC)-stained infarct area compared to control mice, and it has been suggested that although expression levels of $N R 3 A$ are down-regulated in the adult brain, this subunit is still a considerable regulator in a brain under physiological and pathological conditions, such as ischemic stroke. It is hypothesized that strategies that increase NR3A levels in the brain of adult mice could be considered as a target for the development of effective stroke treatment [21]. However, it has been suggested that the expression of neurotransmitter receptors in peripheral blood lymphocytes could parallel that of the brain [9]. The expression of NMDARs in peripheral lymphocytes is induced by inflammatory processes in the brain through neuroendocrine factors [25]. Further studies are needed to provide more confidential information on the NR3A regulatory role in neuroprotective mechanisms.

Several blood biomarkers have been associated after the occurrence of stroke, including MDA and NO. These could be useful in predicting the clinical outcome in patients with ischemic stroke. As in previous studies $[26,27]$, we observed that serum levels of MDA in patients increased within $24 \mathrm{~h}$ after stroke onset. It has been claimed that lipid peroxidation products, such as MDA, are key mediators of neuronal apoptosis induced by oxidative stress [28]. ROS generated during ischemia could initiate toxic changes and react with unsaturated lipids, leading to the generation of several by-products of lipid peroxidation, such as MDA. The production of free radical was elevated in the brain during ischemia, and this process was also detectable in plasma. Generation of free radicals could lead to peroxidation of lipids and oxidation of DNA and proteins, leading to cell membrane damage and activation of calpain-1 as key mediator of neuronal apoptosis. Previous data has demonstrated that the oxidative stress phenomenon can be considered as a pathogenesis factor in ischemic stroke, because the cell membrane in CNS has highly rich polyunsaturated fatty acid side-chains, which are extra vulnerable to an attack of free radicals. Lack of antioxidant enzymes, like glutathione peroxidase and catalase, could be considered as a second cause of oxidative stress injury after ischemia $[29,30]$. Dominguezet et al. demonstrated that an early rise of MDA concentrations has an impact on the clinical outcomes, because patients with the highest MDA concentrations had the worst prognosis in the acute phase $(24 \mathrm{~h}$ and this was associated with NIHSS scoring [30]. Thus, more severe strokes are correlated with higher MDA concentrations [30]. Cano et al. reported significantly decreased NO levels in stroke patients compared with control subjects [31]. Our findings demonstrated a dramatic decrease in NO levels $24 \mathrm{~h}$ and $72 \mathrm{~h}$ after ischemic stroke. This is consistent with previous studies showing that selective inhibition of inducible NO synthase reduces ischemic brain injury in rats [31]. The decrease of NO in plasma after stroke could be a result of eNOS (endothelial NOS) protein hypo activity in the endothelial cells which line blood vessels, and it has been hypothesized that albumin leakage is associated with eNOS and NO reduction in plasma [32]. In conclusion, inflammation leads to a series of cascades which can induce apoptosis. Changes in inflammatory cytokines following ischemic attack could stimulate glutamate receptors, which may lead to alteration of NMDAR subunits. As preliminary findings show, this phenomenon might be a promising diagnostic and therapeutic tool for stroke management. There are some limitations in our study, which should be considered in further studies. For example, the small sample size, lack of evaluation of all NMDAR subunits and lack of gene analysis in protein levels are potential limitations of our project.

Acknowledgments. The authors thank Dr. Vousooghi (Iranian National Center for Addiction Studies, Tehran University of Medical Sciences) for lab accommodation.

Abbreviations. BHT - butylatedhydroxytolune; CNS - central nervous system; CRP - C-reactive protein; CT - computer tomography; EDTA - ethylenediaminetrtraacetic acid; eNOS - epithelial nitric oxide synthase; Frw - forward; GAPDH - glyceraldehyde 3-phosphate dehydrogenase; IL-6 -interleukin 6; 
MDA - malondialdehyde; MCAO - middle cerebral artery; M-MLV - Molony Murine Leukemia Virus; MRI - magnetic resonance imaging; NADPH - nicotinamide adenine dinucleotide phosphate; NIHSS - National Institute of Health Stroke Scale; NMDAR - N-methyl-D-aspartate receptor; NO - nitric oxide; NOX - nitric oxide products; NR - N-methyl D-aspartate recep- tor; NR2A - N-methyl D-aspartate receptor subtype 2A; NR3A - N-methyl D-aspartate receptor subtype 3A; PBS - phosphatebuffered saline; PCR - polymerase chain reaction; ROS - reactive oxygen species; SNSS - Scandinavian Neurological Stroke Scale; TBA - thiobarbituric acid; TNF- $\alpha$ - tumor necrosis factor- $\alpha$; Rev - reverse.

Source of funding: This study was supported by Kashan University of Medical Sciences, Deputy of Research and Technology (Grant No.8946). Conflicts of interest: The authors declare no conflicts of interest.

\section{References}

1. Behdarvandy M, Karimian M, Atlasi MA, et al. Heat shock protein 27 as a neuroprotective biomarker and a suitable target for stem cell therapy and pharmacotherapy in ischemic stroke. Cell Biol Int 2020; 44(2): 356-367.

2. Guo Y, Li P, Guo Q, et al. Pathophysiology and biomarkers in acute ischemic stroke - a review. Trop J Pharm Res 2014; 12: $1097-1105$.

3. Lammerding L, Slowik A, Johann S, et al. Poststroke inflammasome expression and regulation in the peri-infarct area by gonadal steroids after transient focal ischemia in the rat brain. Neuroendocrinology 2015; 103: 460-475.

4. Lee J-M, Grabb MC, Zipfel GJ, et al. Brain tissue responses to ischemia. J Clin Invest 2000; 106(6): 723-731.

5. Wrona D. Neural-immune interactions: an integrative view of the bidirectional relationship between the brain and immune systems. J Neuroimmunol 2006; 172(1-2): 38-58.

6. Boldyrev AA, Carpenter DO, Johnson P. Emerging evidence for a similar role of glutamate receptors in the nervous and immune systems. J Neurochem 2005; 95(4): 913-918.

7. Reiner A, Levitz J. Glutamatergic signaling in the central nervous system: ionotropic and metabotropic receptors in concert. Neuron 2018; 98: 1080-1098.

8. Miglio G, Varsaldi F, Lombardi G. Human T lymphocytes express N-methyl-D-aspartate receptors functionally active in controlling T cell activation. Biochem Biophys Res Commun 2005; 338(4): 1875-1883.

9. Roozafzoon R, Goodarzi A, Vousooghi N, et al. Expression of NMDA receptor subunits in human peripheral blood lymphocytes in opioid addiction. Eur J Pharmacol 2010; 638(1-3): 29-32.

10. Tameh AA, Karimian M, Zare-Dehghanani Z, et al. Role of steroid therapy after ischemic stroke by N-methyl-D-aspartate receptor gene regulation. J Stroke Cerebrovasc Dis 2018; 27(11): 3066-3075.

11. Azami Tameh A, Clarner T, Beyer C, et al. Regional regulation of glutamate signaling during cuprizone-induced demyelination in the brain. Ann Anat 2013; 195: 415-423.

12. Tajalli-Nezhad S, Karimian M, Beyer C, et al. The regulatory role of Toll-like receptors after ischemic stroke: neurosteroids as TLR modulators with the focus on TLR2/4. Cell Mol Life Sci 2019; 76: 523-537.

13. Jin R, Yang G, Li G. Inflammatory mechanisms in ischemic stroke: role of inflammatory cells. J Leukoc Biol 2010; 87(5): 779-789

14. Prass K, Meisel C, Höflich C, et al. Stroke-induced immunodeficiency promotes spontaneous bacterial infections and is mediated by sympathetic activation reversal by poststroke T helper cell type 1-like immunostimulation. J Exp Med 2003; 198(5): 725-736.

15. Vogelgesang A, Grunwald U, Langner S, et al. Analysis of lymphocyte subsets in patients with stroke and their influence on infection after stroke. Stroke 2008; 39: 237-241.

16. Klehmet J, Harms $\mathrm{H}$, Richter $\mathrm{M}$, et al. Stroke-induced immunodepression and post-stroke infections: lessons from the preventive antibacterial therapy in stroke trial. Neuroscience 2009; 158: 1184-1193.

17. Offner $\mathrm{H}$, Subramanian $\mathrm{S}$, Parker SM, et al. Experimental stroke induces massive, rapid activation of the peripheral immune system. J Cereb Blood Flow Metab 2006; 26(5): 654-665.

18. Slowik A, Beyer C. Inflammasomes are neuroprotective targets for sex steroids. J Steroid Biochem 2015; 153: 135-143.

19. Urra X, Cervera Á, Obach V, et al. Monocytes are major players in the prognosis and risk of infection after acute stroke. Stroke 2009; 40: $1262-1268$.

20. Liu Y, Wong TP, Aarts M, et al. NMDA receptor subunits have differential roles in mediating excitotoxic neuronal death both in vitro and in vivo. J Neurosci 2007; 27: 2846-2857.

21. Lee JH, Wei ZZ, Chen D, et al. A neuroprotective role of the NMDA receptor subunit GluN3A (NR3A) in ischemic stroke of the adult mouse. Am J Physiol Cell Physiol 2015; 308(7): C570-C577.

22. Smith CJ, Emsley HC, Gavin CM, et al. Peak plasma interleukin-6 and other peripheral markers of inflammation in the first week of ischaemic stroke correlate with brain infarct volume, stroke severity and long-term outcome. BMC Neurol 2004; 4: 2.

23. Dambinova $\mathrm{S}$, Izykenova $\mathrm{G}$, Gappoeva $\mathrm{M}$, et al. NMDA receptors expression and immunoreactivity in experimental cerebral ischemia and hemorrhage. J Neurochem 2003; 87(S1): 144-144.

24. Gascón S, Sobrado M, Roda JM, et al. Excitotoxicity and focal cerebral ischemia induce truncation of the NR2A and NR2B subunits of the NMDA receptor and cleavage of the scaffolding protein PSD-95. Mol Psychiatry 2008; 13(1): 99-114.

25. Mashkina AP, Cizkova D, Vanicky I, et al. NMDA receptors are expressed in lymphocytes activated both in vitro and in vivo. Cell Mol Neurobiol 2010; 30(6): 901-907.

26. Aygul R, Kotan D, Demirbas F, et al. Plasma oxidants and antioxidants in acute ischaemic stroke. J Int Med Res 2006; 34(4): 413-418.

27. Bir LS, Demir S, Rota S, et al. Increased serum malondialdehyde levels in chronic stage of ischemic stroke. Tohoku J Exp Med 2006; 208(1): 33-39.

28. Aygül R, Kotan D, Yıldırım A, et al. Plasma and cerebrospinal fluid homocysteine, nitric oxide and malondialdehyde levels in acute ischemic stroke: possible role of free radicals in the development of brain injury. Eur J Gen Med 2008; 5(2): 57-63.

29. Yamato M, Egashira T, Utsumi H. Application of in vivo ESR spectroscopy to measurement of cerebrovascular ROS generation in stroke. Free Radical Biol Med 2003; 35: 1619-1631.

30. Domínguez C, Delgado P, Vilches A, et al. Oxidative stress after thrombolysis-induced reperfusion in human stroke. Stroke 2010; 41: $653-660$

31. Cano CP, Bermúdez VP, Atencio HE, et al. Increased serum malondialdehyde and decreased nitric oxide within 24 hours of thrombotic stroke onset. Am J Ther 2003; 10(6): 473-476.

32. Kimoto-Kinoshita S, Nishida S, Tomura TT. Decrease of endothelial nitric oxide synthase in stroke-prone spontaneously hypertensive rat cerebral cortex. Neurosci Lett 2000; 288: 103-106. 
Tables: 1

Figures: 3

References: 32

Received: 13.02 .2020

Reviewed: 3.05 .2020

Accepted: 8.09.2020

Addreses for correspondence:

Abolfazl Azami Tameh, PhD, Assoc. Prof.

Department of Anatomy

School of Medicine

Kashan University of Medical Sciences

Kashan

Post code: 87159-88141

Iran

Tel./Fax: +98 (31) 55621158

E-mail: aazami@kaums.ac.ir

Mohammad Karimian, PhD, Assis. Prof.

Department of Molecular and Cell Biology

Faculty of Basic Sciences

University of Mazandaran

Babolsar

Post Code: 47416-95447

Iran

Tel./Fax: +98 (11) 35302452

E-mail:mdkarimian@gmail.com 
